\begin{tabular}{|l|c|c|l|}
\hline Eiszeitalter und Gegenwart & Band 15 & Seite 81-91 & Öhringen/Württ., 1. November 1964 \\
\hline
\end{tabular}

\title{
Zur Genese der Böden aus Hochflutlehm auf der Niederterrasse im Raume Bonn-Köln-Krefeld
}

\author{
Von ERwIN Kopp \\ Mit 4 Tabellen im Text
}

\begin{abstract}
$\mathrm{Zusammenfassung.} \mathrm{Eingehende} \mathrm{Profiluntersuchungen} \mathrm{von} \mathrm{Böden} \mathrm{aus} \mathrm{den} \mathrm{Hochflut-}$ lehmen der Niederterrasse haben ergeben, daß es sich hier nicht, wie bisher angenommen, um Parabraunerden, sondern um spezifische, außerordentlich stark durch hydromorphe Einflüsse geprägte schichtige Sedimente mit einem Phasenwechsel zum terrestrischen Boden handelt. Für sie wurde der Typenname "Altvega" vorgeschlagen. Es wurden synsedimentäre Tonverlagerungen und Gefügeausbildungen festgestellt, wodurch der Indikatorwert der Tondurchschlämmung eingeschränkt wird.

$\mathrm{S} \mathrm{u} \mathrm{m} \mathrm{m} \mathrm{a} \mathrm{r} \mathrm{y} \mathrm{:} \mathrm{Closer} \mathrm{investigations} \mathrm{of} \mathrm{soil} \mathrm{profiles} \mathrm{developed} \mathrm{out} \mathrm{of} \mathrm{high} \mathrm{flood} \mathrm{loams} \mathrm{of} \mathrm{the}$ Lower Terrace revealed that their classification as "Parabraunerde" (grey-brown podsolic soils) is not correct. It was found that they consist of layered sediments, and having been exposed to strong hydromorphic influences they show an alteration to terrestrial soils. For that great soil group the name "Altvega" is proposed. Synsedimentary clay depositions and structure formations in those soils reduce the indicative value of the clay migration.
\end{abstract}

\section{Einleitung}

Die Bodenbildungen auf der Niederterrasse des Rheins werden, soweit sie aus Hochflutlehm entstanden sind, überwiegend als Parabraunerden, und wo sie aus Flugsanden gebildet wurden, als Sand-Parabraunerde (auch „Parabraunerde aus Sand“ genannt) angesehen (W. PAAS 1961). Bei einer eingehenden Untersuchung einer Parabraunerde-GleyEntwicklungsreihe aus dem Hochflutlehm der Niederterrasse auf ihre Beziehung zwischen dem Bodentyp und dem Gefüge und dem bodentypologischen Wandel innerhalb der Bildungsreihe wurden jedoch hiervon abweichende Ergebnisse erzielt.

Für diese Untersuchungen hatten T. Diez, W. Kerpen und H. Mertens $1961 \mathrm{im} \mathrm{Ge-}$ lände 5 Profile aufgenommen und in ungestörter Lagerung in $35 \times 20 \times 15 \mathrm{~cm}$ großen Blechkästen zur weiteren Bearbeitung in das Institut für Bodenkunde der Universität Bonn gebracht. Es handelte sich dabei um
zwei Parabraunerden aus Hersel bei Bonn und Köln,
zwei Gley-Parabraunerden aus Kapellen II und Vluyn und
einen Parabraunerde-Gley aus Kapellen I.

Eine Orientierung über die Horizontfolge gestattet die Tabelle 1. An diesen Profilen wurden umfangreiche chemische, physikalische, makro-, stereo- und mikroskopische, mineralogische und röntgenanalytische Untersuchungen vorgenommen. Die Untersuchungsergebnisse ließen keine speziellen und allgemein gültigen Beziehungen zwischen Gefüge und Dynamik einerseits und den aufgeführten Bodentypen andererseits erkennen. Bei der Bearbeitung ergaben sich jedoch Merkmale für eine andere Genese dieser Böden. Diese Ergebnisse machen es wahrscheinlich, daß statt der unterstellten Parabraunerde-Dynamik das Auenbodenstadium die Hochflutlehmböden am stärksten geformt hat und sich noch im heutigen Bodenbild dokumentiert.

Die übernommenen, von der Parabraunerde-Dynamik ausgehenden, genetischen Horizontbezeichnungen (Ap- $\mathrm{A}_{3}-\mathrm{B}-(\mathrm{B})$ - usw. Abfolge) werden im folgenden bei der Prüfung der neuen genetischen Situation der Gegenüberstellung wegen bis zur Schlußfolgerung beibehalten. 


\section{Geologische Verhältnisse}

Die Altere (Obere) Niederterrasse wurde vom Beginn der Würm-Vereisung ab angelegt. Spätere Erosionen haben den größten Teil dieser 'Terrasse mit möglichen älteren Bodenbildungen zerstört. In der Jüngeren Dryaszeit (W. PAAS 1961) erfolgte dann die Aufschotterung der über den Bereich der Alteren Niederterrasse hinausgehenden, Laacher Bims führenden Jüngeren Niederterrasse (W. Ahrens 1930). Die Sedimentation auf der Jüngeren Niederterrasse schließt mit einem 1 bis z. T. $5 \mathrm{~m}$ mächtigen Hochflutlehmpaket ab. Der Hochflutlehm wäre demnach erst frühestens während der Jüngeren Dryaszeit, wahrscheinlich später sedimentiert worden. Es hat den Anschein, als ob im Boreal die Aufschlickung der Jüngeren Niederterrasse abklang, denn nach H. NIETsCH (1940) setzte zu dieser Zeit schon eine ungestörte Moorbildung in Rinnen auf der Niederterrasse ein. Weitere Schlickzufuhr und Auflandung nach dieser Zeit ist aber noch für die nördlichen Gebiete denkbar. Das Alter der Bodenbildungen aus Hochflutlehm würde demnach etwa $8000-9000$ Jahre betragen, also relativ jung sein. Die auf der Niederterrasse vorkommenden Dünen sind infolge ihrer Verzahnung mit dem Hochflutlehm wenigstens teilweise etwa gleichaltrig. Nach H. BredDin (1926) steht die Sedimentation des Hochflutlehmes und die Dünenbildung im direkten zeitlichen Zusammenhang mit der Anlage des derzeitigen Hochflutbettes. Die Eintiefung eines Flusses schließt die gleichzeitige Akkumulation im weiten Auenbereich ja nicht aus.

\section{Grundzüge der Bodenentwicklung auf der Niederterrasse}

Die heutige Niederterrasse ist ursprünglich Talboden gewesen; in ihrem oberen, bindigen Teil wurde diese von Hochflutlehmen, d. h. durch periodische Überschwemmungen, aufgebaut. Sie unterscheidet sich in ihrem Entstehungsprinzip kaum von den heutigen Auen. Der Hochflutlehm ist das Produkt der Sedimentationsvorgänge in einer aktiven Aue. Er ist keine Bodenbildung, sondern ein Sediment aus umgelagertem und selektiertem Boden- und Gesteinsmaterial, das aus Suspension mit gewissen faziellen Wirkungen sedimentiert und anfangs besonders den hydromorphen und später den terrestrischen Bodenbildungsfaktoren unterlag.

Für die Hochflutlehme der Niedertermasse kann allgemein die folgende Entwicklung angenommen werden: Sie waren anfangs mindestens an der Basis starken Grundwassereinflïssen ausgesetzt und z. T. Gleyböden (Rohgleye). Mit zunehmender Auflandung bzw. Eintiefung des Rheins ging der permanente Grundwassereinfluß mehr oder weniger stark zurück und die periodischen Überschwemmungen und Grundwasserschwankungen unterwarfen den Hochflutlehm der Auenboden-Dynamik. Die Gley- und Auenphase ist dabei unter den klimatischen und biologischen Verhältnissen des Spät/Postglazials und unter dem Widerspiel von Sedimentation und Bodenbildung zu betrachten. Der periodische Grundwassereinfluß wird sehr weit und lange über die Auflandungsperiode hinaus wirksam gewesen sein und hat möglicherweise Anteil an der Entkalkung. Die Dauer dieser Phase ist nicht bekannt. Die Niederterrasse liegt im Raum Bonn-Köln-Krefeld 8-15 m über dem Rhein, und noch in den letzten Jahrzehnten wurden Rhein-Kegelschwankungen von $10 \mathrm{~m}$ registriert (H. Nietsch 1953). Erst nach dem Ausbleiben der Überschwemmungen und nach einer stärkeren Grundwasserabsenkung werden die Prozesse der terrestrischen Verbraunung voll eingesetzt haben. Den Verhältnissen einer Aue entsprechend, dürften diese hydromorphen und terrestrischen Einflüsse die Niederterrassenböden regional und zeitlich in unterschiedlichem Maße betroffen haben. Die geologische Situation führt aber zu dem Schluß, daß aus dem Hochflutlehm über eine Gleyphase allochthone braune Auenböden entstanden sein müssen, die später durch die terrestrische Verbraunung überprägt wurden. $\mathrm{Ob}$ diese Entwicklung nun weitergehend bis zur Parabraunerde-Dynamik geführt hat, wird noch erörtert. 
Bei den älteren Betrachtungen über die Genese und die Dynamik der Terrassenböden scheinen die Bodenentwicklungsvorgänge auf den Mittelterrassen, besonders aber auf der Hauptterrasse, auf alle Terrassenböden übertragen worden zu sein, ohne daß dabei die Unterschiede zur Niederterrasse im Hinblick auf Sedimentation (dort äolischer Löß, hier Hochflutlehm), Petrographie, Wasserverhältnisse, Alter und den daraus resultierenden unterschiedlichen bodengenetischen Faktoren genügend berücksichtigt worden sind.

\section{Petrographische Verhältnisse}

Die Böden aus Hochflutlehm werden aus mehreren Gründen als Parabraunerden angesehen. Von der im weiteren Raume herrschenden Braunerde-Parabraunerde-Dynamik wird diese Vorstellung leicht abgeleitet. Die sandigere Beschaffenheit der Krume und des darunterliegenden Bereichs sowie die Tondifferenzierung im Profil (s. Tab. 1) scheinen die Parabraunerde-Dynamik zu bestätigen. Hinzu kommen Tonabsonderungen auf Klüften und in Poren, so daß die Einstufung als Parabraunerden hinreichend begründet erscheint.

Tabelle 1:

Horizontierung, Tongehalt und Feinsand-: Grobsandverhältnis (FS : GS) von Böden aus Hochflutlehm

\begin{tabular}{|c|c|c|c|c|c|c|c|c|c|c|c|}
\hline \multicolumn{4}{|c|}{$\begin{array}{c}\text { Hersel (Bonn) } \\
\text { Parabraunerde }\end{array}$} & \multicolumn{4}{|c|}{$\begin{array}{l}\mathrm{K} \text { ö } 1 \mathrm{n} \\
\text { Parabraunerde }\end{array}$} & \multicolumn{4}{|c|}{$\begin{array}{c}\text { V1 u y n } \\
\text { Gley-Parabraunerde }\end{array}$} \\
\hline Hor. & $\mathrm{cm}$ & $\%$ Ton & FS:GS & Hor. & $\mathrm{cm}$ & $\%$ Ton & FS:GS & Hor. & $\mathrm{cm}$ & $\%$ Ton & FS:G \\
\hline Ap & 25 & 14,6 & 2,5 & Ap & 21 & 15,1 & 3,5 & Ap & 19 & 13,4 & 2,7 \\
\hline$A_{3}$ & 41 & 22,6 & 1,0 & $\mathrm{~A}_{3}$ & 36 & 22,2 & 6,3 & $\mathrm{~A}_{3}$ & 30 & 24,4 & 4,8 \\
\hline B & 62 & 25,8 & 5,9 & $\mathrm{~A}_{3} \mathrm{~B}$ & 55 & 29,9 & 7,8 & GB & 46 & 24,0 & 6,1 \\
\hline $\mathrm{B}(\mathrm{B})$ & 82 & 23,9 & 3,5 & B & 82 & 30,8 & 24,0 & BGo & 64 & 26,6 & 4,2 \\
\hline (B) & 106 & 20,4 & 2,8 & (B) & 92 & 29,8 & 13,6 & Go & 82 & 20,2 & 1,5 \\
\hline $\mathrm{DCa}$ & 135 & 5,5 & 2,5 & (B) $\mathrm{CCa}$ & 98 & 19,6 & 8,4 & Gor & 100 & 24,6 & 2,9 \\
\hline & & & & (B)CCa & 114 & 15,5 & 4,4 & GorCa & 107 & 17,1 & 3,5 \\
\hline & & & & $\mathrm{DCa}$ & 131 & 1,6 & 9,7 & DGr & 130 & 8,2 & 4,7 \\
\hline
\end{tabular}

\begin{tabular}{|c|c|c|c|c|c|c|c|}
\hline \multicolumn{4}{|c|}{$\begin{array}{c}\text { K a pelle n I I } \\
\text { Gley-Parabraunerde }\end{array}$} & \multicolumn{4}{|c|}{$\begin{array}{c}\text { Ka pelle n I } \\
\text { Parabraunerde-Gley }\end{array}$} \\
\hline Hor. & $\mathrm{cm}$ & $\%$ Ton & FS:GS & Hor. & $\mathrm{cm}$ & $\%$ Ton & $\mathrm{FS}: \mathrm{GS}$ \\
\hline Ap & 30 & 13,4 & 2,2 & Ap & 21 & 15,1 & 1,8 \\
\hline$A_{31}$ & 50 & 18,1 & 3,9 & $\mathrm{GA}_{3}$ & 36 & 15,2 & 2,4 \\
\hline $\mathrm{A}_{32}$ & 60 & 26,6 & 8,4 & $\mathrm{~A}_{3} \mathrm{G}$ & 48 & 21,7 & 3,0 \\
\hline $\mathrm{GoB}_{11}$ & 70 & 28,6 & 8,2 & BGo & 82 & 33,0 & 7,5 \\
\hline $\mathrm{GoB}_{12}$ & 92 & 26,7 & 8,1 & Gro & 110 & 28,2 & 4,4 \\
\hline BGo & 136 & 28,3 & 7,5 & DGro & 140 & 15,0 & 49,0 \\
\hline DGo & 150 & 19,1 & 2,6 & & & & \\
\hline
\end{tabular}

Der Hochflutlehm der Niederterrasse besteht zum großen Teil aus umgelagertem Lößmaterial und anderen tonigen, schluffigen und sandigen Verwitterungsprodukten der stromauf liegenden Einzugsgebiete. Die petrographische Zusammensetzung und der vertikale Aufbau der Terrassen und Auen ist von der ständig wechselnden Transportkraft des Flusses und der Überflutungswässer abhängig. In gefällereichen Talabschnitten großer Flüsse ist von vornherein kein einheitlicher petrographischer Aufbau der Terrassen bzw. der Talauen zu erwarten.

Die Auswertung der Kornanalysen und der stereomikroskopischen Beobachtungen erbrachten starke Zweifel an einem petrographisch einheitlichen Aufbau innerhalb der einzelnen Profile. Es wurden vielmehr eine mehrschichtige Zusammensetzung der Böden aus Hochflutlehm festgestellt. 
Bei der Feldansprache sind mit der Fingerprobe bei möglicherweise schichtigen Böden kaum genetische Deutungen möglich. Zudem läßt ein unterschiedlicher Tongehalt nur bei einheitlichem Ausgangsmaterial genetische Schlüsse zu. Deshalb kann erst unter Einbeziehung der Sandfraktionen festgestellt werden, wieweit das Bodenprofil petrographisch homogen ist. Die Sandfraktion als relativ stabiler Bestandteil verschiebt sich bei gleichem Ausgangsmaterial innerhalb eines Profils durch die Verwitterung kaum in ihrem Verhältnis von z. B. Feinsand zu Grobsand. Wechselnde Feinsand-Grobsand-Verhältnisse zeigen deshalb primär unterschiedliche petrographische Zusammensetzungen an. In der Tab. 1 sind in einer Spalte neben dem Tongehalt die Feinsand: Grobsand-Verhältnisse (FS : GS) aufgeführt. Die Ubberprüfung der 5 Profile ergibt folgende Situation:

Alle Profile zeigen von Horizont zu Horizont stark wechselnde Feinsand : GrobsandVerhältnisse, die keine Beziehung zum Tongehalt zeigen und damit eindeutig den mehrschichtigen Aufbau der Hochflutlehmböden nachweisen. Innerhalb der Profile schwankt das Feinsand : Grobsand-Verhältnis folgendermaßen:

Tabelle 2:

Schwankungsbreite der Feinsand : Grobsand-Verhältnisse

\begin{tabular}{|c|c|c|c|c|c|}
\hline Profil: & Hersel & $\mathrm{K} \ddot{\mathrm{l}} \ln$ & Vluyn & $\mathrm{K}$ a pellen I I & $\mathrm{K}$ a peller \\
\hline & $\begin{array}{c}1-5,9 \\
1\end{array}$ & $\begin{array}{c}3,5-24,0 \\
1\end{array}$ & $\begin{array}{c}1,5-6,1 \\
1\end{array}$ & $\begin{array}{c}2,2-8,4 \\
1\end{array}$ & $\begin{array}{c}1,8-7,5 \\
1\end{array}$ \\
\hline
\end{tabular}

Im Profil Köln zeigen z. B. die einzelnen Horizonte folgende Tongehalte und Feinsand : Grobsand-Verhältnisse:

Tabelle 3

Tongehalt zum Feinsand : Grobsand-Verhältnis

\begin{tabular}{ccc} 
Horizont & Tongehalt & Feinsand: Grobsand \\
\hline $\mathrm{A}_{3} \mathrm{~B}$ & $29,9 \%$ & 7,8 \\
$\mathrm{~B}$ & $30,8 \%$ & 24,0 \\
(B) & $29,8 \%$ & 13,6
\end{tabular}

Bei annähernd gleichem Tongehalt weisen die drei Horizonte ganz verschiedene Sandbeimengungen auf, die nur auf die primär unterschiedliche Ablagerung zurückgeführt werden können. Der fast gleich hohe Tongehalt dieser übereinander liegenden Schichten ist praktisch zufällig. Dagegen könnten am Profil Kapellen II die Tongehalte und die Feinsand: Grobsand-Verhältnisse des $\mathrm{A}_{32}, \mathrm{GoB}_{11}$ und $\mathrm{GoB}_{12}$ auf eine mehrhorizontige Schicht schließen lassen. Die Betrachtung der gesamten Analyse läßt aber ebenfalls eine echte Mehrschichtigkeit erkennen.

Tabelle 4

\begin{tabular}{lccccccc}
\multicolumn{7}{c}{ Feinsand : Grobsand-Verhältnis zur Schichtung } \\
& $(4+5: 6)$ & 1 & 2 & 3 & 4 & 5 & 6 \\
Horizont & FS : GS & $<0,002$ & $0,002-0,006$ & $0,006-0,002$ & $0,002-0,06$ & $0,06-0,2$ & $0,2-2,0$ mm Fraktion \\
\hline$A_{32}$ & 8,4 & 26,6 & 11,3 & 18,7 & 25,2 & 13,6 & 4,6 \\
GoB $_{11}$ & 8,2 & 28,6 & 9,2 & 15,5 & 21,7 & 19,9 & 5,1 \\
GoB $_{12}$ & 8,1 & 26,7 & 8,0 & 12,9 & 18,0 & 28,6 & 5,8
\end{tabular}

Trotz ähnlicher Tongehalte und beieinanderliegender Verhältniszahlen ist aus den nach unten ansteigenden Sandanteilen die Heterogenität zu ersehen. Die quantitativen Proportionen müssen darum ebenfalls geprüft werden.

Bei der Bestimmung der Körnung hat jede Kornanalyse den Nachweis für eine selbständige Schicht erbracht. $\mathrm{Da}$ die petrographische Schichtung der fluviatilen Sedimente aber kaum mit der genetischen Horizzonteinteilung zu parallelisieren ist, wird das petrogra- 
phische Bild durch eine Probe je Horizont nur unvollständig wiedergegeben. Wie Vergleiche ergaben, wechselt die Textur auch innerhalb der genetischen Horizonte beträchtlich. Auffällig ist, daß dabei die Fein- und Grobsandanteile (FS : GS) wesentlich stärker betroffen werden als der Tongehalt.

Um ganz sicher Verwitterungseinflüsse auszuschalten, sollten die resistenten Quarzanteile der Sandfraktionen zueinander ins Verhältnis gesetzt werden (P. KundLER 1959). Der sog. "Quarztest" setzt eine mineralogische Untersuchung voraus und ist bei feineren Profilunterschieden notwendig. Stärkere Unterschiede, wie sie hier auftreten, sind aus der Korngrößenanalyse am Verhältnis Feinsand : Grobsand auch unmittelbar abzulesen. Der Quarztest wurde in Anlehnung an die Kundlersche Silikatzahl-Bestimmung (P. KundLER 1956) an 3 Profilen vorgenommen. Es traten etwas andere Verhältniszahlen auf. Die Mehrschichtigkeit wird aber auch hierbei deutlich bestätigt.

Die anscheinend weit verbreitete sandige Krume bzw. obere Schicht stellt ein besonderes Problem dar. Die Feinsand : Grobsand-Verhältnisse charakterisieren ein relativ grobsandreiches Material. Dazu kommt eine deutliche Begrenzung der Stein- und Kiesführung auf die sandigere Oberschicht, die an ihrer Basis in einer Kiesgrube bei Hersel sogar durch eine Steinsohle vom bindigeren Hochflutlehm getrennt wird. Diese sandigeren Deckschichten stellen wahrscheinlich eine jüngere stratigraphische Einheit dar, die durch ihre beachtliche Erstreckung (Bonn-Köln-Krefeld) anscheinend mit einer jungen Erosions-Sedimentationsphase des Rheins zusammenhängt. Daneben kann sicher auch mit Flugsandeinwehungen und Tonausblasungen im ganzen Niederterrassenbereich gerechnet werden, was dann ebenfalls zu einem absolut oder relativ geringeren Tonanteil des Oberbodens beigetragen haben wird. Diese Vorgänge würden auch die stellenweise auffällige Beschränkung der sandigeren Oberschicht auf den Ap-Horizont, die gepflügte Krume, erklären.

Die petrographischen Untersuchungsergebnisse schließen die Einstufung als Parabraunerde auf Grund petrographischer Merkmale aus. Die Bestimmung der Parabraunerde aus der Texturdifferenzierung kann, soweit nicht andere eindeutige Merkmale vorliegen, bei schichtigen Böden nicht allein durch die Berücksichtigung der Fingerprobe und des analytisch ermittelten Tongehaltes vorgenommen werden. Es müssen dazu die gesamten Kornfraktionen herangezogen und dabei die absoluten und relativen Anteile besonders der restistenten Korngrößen und Minerale ausgewertet werden.

Die Mehrschichtigkeit der Böden aus Hochflutlehm auf der Niederterrasse des Raumes Bonn-Köln-Krefeld dürfte als sicher gelten.

\section{Tonverlagerung}

Die Parabraunerde-Dynamik ist durch eine abwärts gerichtete Tonverlagerung gekennzeichnet. Diese führt durch die Tonverarmung der oberen Horizonte und Tonanreicherung im unteren Profilteil zu einer Texturdifferenzierung des Profils.

Eine gleichsinnige Texturabfolge wurde auch bei den Hochflutlehmen angetroffen und hatte mit dazu beigetragen, diese Böden bodentypologisch der "Parabraunerde" zuzuordnen. Diese Art der Tonverlagerung und ihre Erscheinungen, besonders die Tonüberzüge auf den Kluftfächen, werden allgemein als spezifisches Merkmal der ParabraunerdeDynamik angesehen.

\section{a. Tonverlagerung im Hochflutlehm}

Bei den Hochflutlehmen ergibt sich die Notwendigkeit, den Zeigerwert der Tonverlagerung für die Klassifizierung einzuschränken, denn mit der Sedimentation des Hochflutlehmes und der Auenboden-Dynamik sind intensivste Durchschlämmungen gekoppelt, die auch die morphologischen Merkmale der Durchschlämmung erzeugen. 
Im allgemeinen erfolgt die Auflandung in der Aue aus vielen und verschiedenartigen Suspensionen. Die ständigen Überschwemmungen und das abwechselnde Trockenfallen bewirken eine außerordentlich starke, nach unten gerichtete, fortlaufende Durchspülung des gesamten aufgelandeten Materials. Dazu kommen aber noch aufwärts und abwärts gerichtete Grundwasserspiegelschwankungen und oft eine zügige seitliche Perkonlation. Je nach der Höhe der Aue über dem niedrigsten Grundwasserspiegel werden diese verschieden gerichteten Wassereinflüsse mit unterschiedlicher Intensität wirksam. Es ist aber nicht anzunehmen, daß diese Durchspülung andere mechanische Wirkungen auf die feinsten Bodenteilchen hat als die Niederschläge. Es wird gerade bei der Durchspülung in der Aue mit stärkeren Tonverlagerungen zu rechnen sein als in terrestrischen Böden. Im Gegensatz zur Parabraunerde wird die Tonverlagerung nicht erst nach der Verbraunung, sondern unmittelbar mit der Auflandung, also syngenetisch, beginnen. Diese Durchschlämmung müßte eine dem allochthonen (braunen) Auenboden inhärente Eigenschaft sein, die allerdings wegen der in einer Aue unterschiedlich wirkenden Bodenbildungsfaktoren beträchtlich varieren kann.

Der Tongehalt des Hochflutlehmes stammt sicher zum größten Teil aus dem Bodenmaterial der stromauf im Einzugsgebiet liegenden Lößböden. Es wurden hier also ortsund typenfremde Bodentonminerale abgelagert. Ferner ist für die Jüngere Dryaszeit und vielleicht auch für das Boreal in der Aue nur eine spärliche Vegetation anzunehmen. Das würde jeweils nach dem Trockenfallen der Aue bei der nächsten Austrocknung zu Schrumpfungen und Trockenrißbildungen geführt haben. Diese Risse wären dann Ableitungsbahnen für die nächsten Niederschläge und Hochwässer (mit Tonsuspensionen) gewesen; zum anderen führten sie zu einer vertikalen Gefügegliederung, nämlich zum polyedrischprismatischen Gefüge des Sedimentes. Die Tondurchschlämmung und auch die Gefügeausbildung vollziehen sich schon von der Basis des Hochflutlehmes an und sind hier sedimentationseigene Kriterien und nicht nur Wirkungen der Bodenbildung.

b. Mikromorphologie

Die Tonverlagerung wurde bei den 5 Profilen an 45 Dünnschliffen, die aus den Gefügehorizonten stammen, untersucht.

Die Bearbeitung erbrachte übereinstimmend, daß die Ap- und $\mathrm{A}_{3}$-Horizonte, soweit sie mit der oberen sandigeren Deckschicht zusammenfallen, nicht das typische Bild des verarmten $\mathrm{A}_{3}$-Horizontes bieten. Der gesamte unterlagernde, bindige Hochflutlehm weist mikromorphologisch einen einheitlichen Charakter auf. Mikromorphologisch unterscheiden sich die B-, B(B)-, (B)-, GoB-, BGo-, BC-, GCa- und andere Horizonte kaum voneinander. Sie weisen in der Parabraunerde, Gley-Parabraunerde und z. T. auch im ParabraunerdeGley etwa den gleichen Anteil und die gleiche Art der Verteilung der doppelbrechenden, orientierten Tonsubstanz bis in die Profilbasis auf. Besonders auffällig ist die Tatsache, daß in allen Profilen, unabhängig von der Parabraunerde-Horizontierung, das derzeitige Kluftund Porensystem nur in geringem Maße jüngere, aktuelle Tonverlagerungsprozesse erkennen läßt (K. ZrmmermanN 1962).

Die mit der Horizontbezeichnung der Parabraunerde ausgedrückten genetisch-dynamischen Verhältnisse wurden mithin nicht bestätigt, sondern die Gleichartigkeit des mikromorphologischen Bildes über das ganze Profil zeigt nicht $\mathrm{A}_{3^{-}}, \mathrm{B}-, \mathrm{B}(\mathrm{B})-$, (B)C- oder BG-, sondern M-Horizonte an.

Mikromorphologische Vergleichsuntersuchungen rezenter Auenböden fehlen leider noch überall. Sieht man von dem Fehlen der aktuellen Durchschlämmung ab, so bietet sich beim Hochflutlehm im einzelnen Dünnschliff das Bild eines durchschlämmten Bodens (W. Kubiena 1963), das bislang der Parabraunerde-Dynamik gleichgesetzt wurde. Wenn aber die mechanische Tondurchschlämmung der Parabraunerde zu Fließstrukturen be- 
stimmter Tonsubstanzen führt, muß auch die syngenetische mechanische Durchschlämmung bei der Sedimentation dasselbe mikromorphologische Bild erzeugen. Auch die Tonhäutchen der Aggregate besitzen bei fluviatilen Sedimenten (möglicherweise auch beim Schwemmlöß und bei Fließerde) nur bedingten Zeigerwert für die ParabraunerdeDynamik.

\section{c. Tonverlagerung in den Schottern und Sanden}

In den unverwitterten Schottern und Sanden der Niederterrasse treten in Abständen von $20-100 \mathrm{~cm}$ häufig bis in über $10 \mathrm{~m}$ Tiefe rotbraune, lehmige Bänder auf, die nicht immer schichtenparallel verlaufen und deshalb auf sekundäre Einlagerung hinweisen. W. PAAs (1961) führt das Auftreten der rötlichbraunen, lehmigen Bänder innerhalb der Schotter und Sande auf Tonverlagerungen rezenter Bodenbildungsprozesse zurück und sieht diese als Nachweis für die Parabraunerde-Dynamik an.

Es läßt sich so aber der Tonnachschub bis in mehrere Meter Tiefe für zahlreiche lehmige Tonbänder mengenmäßig, z. B. aus einer nur lehmigen Sand-Parabraunerde, nicht erklären. Einmal ist kaum zu erwarten, daß bei diesen relativ jungen, sandigen Böden derartige Tonmengen produziert werden können. Zum anderen wäre die Art der Ausfällung kaum verständlich. Man kann annehmen, daß die Fällung oder Stauung wandernder Tonteilchen in Horizonten mit anderen physiko-chemischen Potentialen bzw. anderen mechanischen Rückhaltevermögen erfolgt. Es wäre nicht erklärbar, wie abwandernde Tonteilchen zahlreiche Flockungs- oder Filterschichten gleichartiger Tonhaltefähigkeit ungehindert passieren können, um dann in mehreren Metern Tiefe festgelegt $\mathrm{zu}$ werden. Wahrscheinlicher wäre doch die Festlegung aller Tonteilchen bereits in der ersten Schicht mit einem Tonhaltevermögen, wie es normalerweise bei bindigen Böden in den dem Tonverarmungshorizont $\mathrm{A}_{3}$ unmittelbar folgenden Tonanreicherungshorizont B geschieht. Bei einer Auflösung des B-Horizontes nach unten, wie es W. PAas (1961) ansieht, wäre in durchlässigen Böden bei terrestrischer Bodendynamik ein baldiges $\mathrm{Ab}$ klingen der Toneinschlämmungsbänder zu erwarten, und nicht eine bis über $10 \mathrm{~m}$ Tiefe reichende, unverminderte intensive Tonbänderung.

Sieht man die Entstehung der Tonbänderung als Produkt der spezifischen Wasser- und Bodenverhältnisse in einer Aue an, so lassen sie sich zwanglos als Tondurchschlämmungssäume versickernder Hochwässer erklären, die wahrscheinlich im Gegenspiel von Einsickerung und Grundwasseranstieg und -abstieg an primär bedingten (petrographisch) oder dadurch geschaffenen Unstetigkeitslinien (Go) durch Konzentrierung niedergeschlagen wurden. Sie können auch einfach Tonüberzüge der Schotter aus abklingenden Überschwemmungen darstellen. Danach würde die Entstehung der tonigen Bänder zeitlich mit dem Aufbau der Terrasse zusammenfallen können, da toniges Verwitterungsmaterial zu allen Zeiten herangeführt wurde. Das Auftreten der tonigen Bänder setzt keine überlagernden lehmigen Böden oder gar Parabraunerden voraus.

\section{Tonmineralzusammensetzung}

Das Vorhandensein spezifischer Tonminerale oder Tonmineral-Gemeinschaften kennzeichnet unter Berücksichtigung des Ausgangsmaterials bestimmte Verwitterungsprozesse oder dynamische Vorgänge im Boden.

Von 4 Profilen wurden zur Orientierung insgesamt 29 Pulverdiagramme angefertigt und röntgenographisch untersucht. Die Tonmineral-Garnituren setzen sich durchweg aus einem überwiegenden Anteil illitischer, einer zurücktretenden Menge aufweitbarer Illite und einem bedeutenden Gehalt kaolitischer Tonminerale zusammen.

Die Profile weisen danach in sich vom Ap- bis zum C-Horizont die gleiche Tonmineralzusammensetzung auf und die Tonmineral-Garnitur aller 4 Profile, von der Parabraun- 
erde bis zum Parabraunerde-Gley, ist untereinander gleich. Die Röntgenanalyse bestätigt die übrigen Untersuchungen und spricht mit der Gleichartigkeit der Tonmineralzuzsammensetzung in den verschiedenen Profilen weit eher für Auenboden- als für ParabraunerdeDynamik.

Die Tatsache, daß die Tonmineral--Garnitur der tonigen Bänder in den Schottern sich nicht von der des überlagernden Bodens unterscheidet (W. PAAS 1961), spricht nicht zwingend für die Herkunft aus der Parabraunerde. Die Tonsubstanz der Bänder und des Hochflutlehmes stammen überwiegend aus den Verwitterungsprodukten orts- und typfremder Böden und befinden sich hier auf sekundärer oder sogar auf tertiärer Lagerstätte. Die Einheitlichkeit der Tonmineral-Zusammensetzung kann dagegen auch als Beweis dafür angesehen werden, daß die Tonsubstanzen in den Bändern und im darüber liegenden Boden ihre Zusammensetzung bisher kaum verändert haben und deshalb mindestens die Einflüsse der terrestrischen Verwitterung sehr gering sind.

\section{Gefüge der Böden aus Hochflutlehm}

Die Bodentypen und ihre Horizonte drücken ihre petrographisch-dynamische Eigenart in bestimmten Gefügearten aus. Bei den 5 Profilen konnten die Ergebnisse der makroskopischen und stereomikroskopischen Gefügeuntersuchungen nicht mit den angegebenen Bodentypen in Einklang gebracht werden. Die Gefügeausbildung schwankt zwischen Subpolyedern und Prismen. Die allgemeine Tendenz geht von Subpolyedern in den oberen Horizonten zum polyedrisch-prismatischen Gefüge in den B-, (B)-, G- und (B)C-Horizonten. Dabei wurde häufig innerhalb der genetischen Horizonte eine Gefügewandlung festgestellt, auch wiesen dieselben Horizonte vergleichbarer Bodentypen recht unterschiedliche Gefüge auf. Ferner unterscheidet sich das Gefüge des rezenten Gleyes mit seinen Go- und Gor-Horizonten kaum von den B(B)-, (B)- und (B)C-Horizonten der Parabraunerden. Das prismatisch-polyedrische Gefüge widerspricht jedoch völlig dem Wesen des (B)-Horizontes und spricht gegen die Braunerde-Parabraunerde-Dynamik in den Hochflutlehmen. Das sich durch den gesamten Hochflutlehm ziehende polyedrische und prismatische Gefüge stellt sich deutlich als Relikt und Nachweis der semiterrestischen Phase dar.

Bei der Horizontierung der Profile ist nicht nur die Texturdifferenzierung entscheidend, sondern auch das Gefüge der einzelnen Horizonte. Bei den Hochflutlehmen sind Tongehalt, Gefüge und Tondurchschlämmung primärer und interstrataler Natur und nicht das Produkt der Parabraunerde-Dynamik. Es sind die Gefüge durchschlämmter Schichten, wobei die Durchschlämmungserscheinungen jedoch nicht das Ergebnis aktueller, sondern synsedimentärer Prozesse sind.

\section{Silikatverwitterung}

Durch die Bestimmung der Silikatanteile in den verschiedenen Sandfraktionen (P. Kundler, 1956, E. Kopp 1961) wurde versucht, die bei einer Parabraunerde-Verwitterung zu erwartende Silikatverarmung oder die stärkere Silikatzerstörung in der feinsten Sandfraktion der Ap- und $\mathrm{A}_{3}$-Horizonte festzustellen.

Dieser Nachweis konnte nicht erbracht werden. Im Gegenteil wurde mehrmals in der sandigeren Deckschicht ein höherer Silikatgehalt gefunden als in den verwitterungsextensiven (B)-Horizonten. Ein für die Parabraunerde spezifischer Silikatabbau konnte nicht beobachtet werden. Damit wurde wiederum die Mehrschichtigkeit und das Fehlen einer ausgeprägten Parabraunerde-Dynamik wahrscheinlich gemacht.

\section{Gehalt an Eisenkonkretionen}

Die Behauptungen über die Dominanz der semiterrestrischen Phase in den Hochflutlchmen konnten durch Feststellung ungewöhnlich großer Mengen von Eisenkonkretionen 
erhärtet werden. In der sandigeren Deckschicht wurden meist abgerollte, harte Konkretionen vorgefunden, während sie im bindigeren Hochflutlehm weicher und rauhflächig waren.

Das Profil Köln (Parabraunerde) hat z. B. mit 40-65\% Eisenkonkretionen in der Fraktion 0,5-1,0 $\mathrm{mm}$ einen ungewöhnlich hohen Gehalt, der etwa dem des rezenten Parabraunerde-Gleyes Kapellen I gleichkommt. Die Konkretionsanteile weisen zum anderen keine Beziehungen zur genetischen Horizontierung auf. $A_{3}, B,(B)$ und (B)C enthalten als Eluvial-, Illuvial- und Verwitterungshorizonte ohne nennenswerte Stoffzufuhr einen nach unten ansteigenden Konkretionsgehalt, der nicht zur Parabraunerde-Dynamik paßt. Diese Konkretionen können nicht aus der Parabraunerde-Dynamik herrühren, sondern stellten sich hier deutlich als Relikte der früheren Gley- und Auenboden-Phase dar.

\section{Entkalkung}

Nach W. PAAS (1961) befinden sich allochthone Kalkauenböden im Bereich des derzeitigen Hochflutbettes. Durch die Überschwemmungen des Rheins wurde sicher auch den Böden der Jüngeren Niederterrasse Kalziumkarbonat zugeführt. Dieses ist heute aus dem oberen Teil der Profile entfernt und erst in $0,90 \mathrm{~m}$ oder auch unter 1,50 m Tiefe noch anzutreffen. Zweifellos haben die Hochflutlehme eine Entkalkung und Verbraunung erfahren. W. KubIENA (1953) weist aber darauf hin, daß die Entkalkung der braunen Auenböden durch starke Auswaschung relativ schnell vor sich gehen kann. Die Dauer der Entkalkungs- und Verbraunungszeit sind nicht bekannt. Die teilweise Entkalkung und eine Verbraunung sind als Einfluß der terrestrischen Bodenbildungsfaktoren zu beachten und dürfen sich in einer schwachen Überprägung der Böden aus Hochflutlehm auswirken.

\section{Schlußfolgerungen}

Die vielseitigen Untersuchungen von 5 Profilen aus Hochflutlehm lassen erkennen, daß diese Böden aus mehrschichtigen, fluviatilen Sedimenten mit synsedimentärer Tondurchschlämmung und Gefügeausbildung unter starkem hydromorphen Einfluß entstanden, und daß sekundär nur eine schwache Verbraunung erfolgte.

1. Horizontbezeichnung und $T y p$

Die scheinbaren Merkmale der Parabraunerde-Dynamik werden als Folge der Sedimentation und der Gley- und Auenboden-Phase gedeuter. Aus der Zusammenschau der vorliegenden Resultate könnte man geneigt sein, diese Böden folgendermaßen anzusprechen:

Mehrschichtige, allochthone braune Auenböden (Vega) mit schwacher Verbraunung und teilweise unterschiedlichen Grundwassereinflüssen.

Die Horizontbezeichnung der Profile würde aus dieser Sicht folgende Änderungen erfahren:

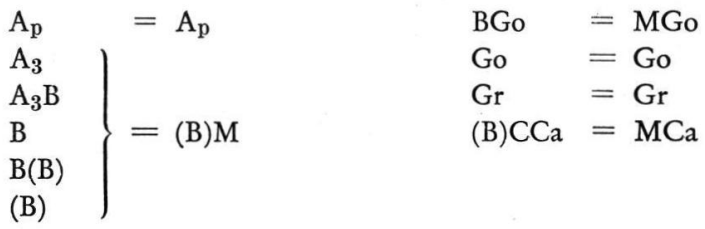

Nach den Prinzipien der Systematik dürften die grundwasserfreien Böden allerdings nicht als Auenböden bezeichnet werden, weil Grundwasser- und Überschwemmungseinflüsse bereits lange fehlen. Die höher liegenden Böden weisen rein terrestrische Perkolation auf. Andererseits sind die Braunerdemerkmale noch zu gering, um diese Böden der Braunerde-Klasse zuzuordnen. Die semiterrestrische Phase, 
obwohl bereits lange zurückliegend, beherrscht noch heute das Bodenbild. Es sind aber trotzdem terrestrische Böden, ohne daß ihre Morphologie und ihr Charakter den Braunerden oder Parabraunerden entsprechen.

Die spezifischen Bodenbildungsprozesse, nachgewiesen durch ihre genetische, petrographische und gefügekundliche Eigenart sowie der heute vor sich gehende Wandel der Dynamik dieser Böden würde im Sinne der Bodensystematik (E. MüCKENHAUSEN, 1962) eine Ausscheidung als besonderen terrestrischen Bodentyp rechtfertigen.

Es läge nahe, die grundwasserfernen Böden aus Hochflutlehm als „Terrassenböden“ zu bezeichnen und sie von den typischen Auenböden und den Braunerden abzugrenzen. Es ist aber vorstellbar, daß ein großer Teil der stromf ernen und eingedeichten Auenböden eine ähnliche Bodenbildung aufweist und mit in diesen Typ einbezogen werden könnte. Dann würden die Bezeichnungen "Altvega" besser geeignet erscheinen. Mit diesen Begriffen wären ihre Entstehung, ihre Wandlung und auch ihre morphologischen und geographischen Merkmale gut gekennzeichnet.

Die „Parabraunerden“ von Hersel und Köln würden danach als Braunerde-Altvega, die "Gley-Parabraunerde" von Kapellen II als

Braunerde-Altvega,

die "Gley-Parabraunerde" von Vluyn und

der "Parabraunerde-Gley" von Kapellen I als Auengley

anzusprechen sein.

\section{Tondurchschlämmung der Aue}

Es gibt eine synsedimentäre Tondurchschlämmung und eine Gefügeausbildung fluviatiler Sedimente, die bei der intensiven Durchspiilung von tonigem Material etwa das Bild einer durchschlämmten Parabraunerde bieten, ohne das Ergebnis irgendeiner Bodendynamik zu sein. Damit wird der bisherige Zeigerwert der morphologischen und mikromorphologischen Tondurchschlämmung als Nachweis der Parabraunerde-Dynamik für Böden aus fluviatilen und möglicherweise auch aus anderen umgelagerten Sedimenten stark eingeschränkt.

3. Alter

Die Bodenbildungen auf dem holozänen Hochflutlehm sind höchstens 9000 Jahre alt und haben eine neue Deutung erhalten. Demnach hat hier in diesem Zeitraum keine Parabraunerde-Bildung stattgefunden.

\section{$\mathrm{Schriften-Nachweis}$}

Ahrens, W.: Die Trennung der „Niederterrasse“ am Mittel- und Niederrhein in einen diluvialen und einen alluvialen Teil auf Grund der Geröllführung. - Z. deutsch.-geol. Ges. 82, S. 129-141, Berlin 1930.

Breddin, H.: Löß, Flugsand und Niederterrasse am Niederrhein. - Jb. preuß. geol. L.-A. 46 (1925), S. 635-662, Berlin 1926.

Kopp, E.: Besonderheiten der intrasedimentären Verwitterung miozäner Ablagerungen der Niederlausitz. - Ber. d. geol. Gesell. 6, S. 216, Berlin 1961.

KubienA, W.: Bestimmungsbuch und Systematik der Böden Europas. - Enke, Stuttgart 1953. - Mündliche Mitteilung, 1963.

Kundler, P.: Beurteilung forstlich genutzter Sandböden im norddeutschen Flachland. - Archiv f. Forstw. 5, S. 585, Berlin 1956. - - Zur Methodik der Bilanzizerung der Ergebnisse von Bodenbildungsprozessen (Profilbilanzierung), dargestellt am Beispiel eines Texturprofils auf Geschiebemergel in Norddeutschland. - Z. Pflanzenernähr., Düngung etc. 86 (131), S. 215, Weinheim 1959.

MüCKenhausen, E.: Entstehung, Eigenschaften und Systematik der Böden der Bundesrepublik Deutschland. - DLG-Verlag, Frankfurt M. 1962. 
Nietsch, H.: Pollenanalytische Untersuchungen auf der Niederterrasse bei Köln. - Z. deutsch. geol. Ges. 92, S. 350, Berlin 1940. - - Zur spät- und nacheiszeitlichen Entwicklung einiger Flußtäler im nordwestlichen Deutschland. - Z. deutsch. geol. Ges. 104, S. 29-40, Hannover 1953.

PAAs, W.: Rezente und fossile Böden auf niederrheinischen Terrassen und deren Deckenschichten. Eiszeitalter u. Gegenwart 12, S. 165, 1961.

ZimmermanN, K.: Mündliche Mitteilung, 1962.

Manuskr. eingeg. 27. 3. 1963.

Anschrift des Verf.: Dr. Erwin Kopp, Institut für Bodenkunde der Universität, Bonn, Nußallee 13. 